\title{
Mobility of Ions in Gases
}

Hajime TANUMA and Shiro MATOBA* (首都大学東京 田沼 肇, 的場 史朗*)

Tokyo Metropolitan University Kazumasa OHTSUKI (電気通信大学 大瞡一雅) The University of Electro-Communications * present address : Rikkyo University 
Outline

1. Introduction to lon mobility in gas

Experiments

\section{Theory}

2. Closed-shell systems

Experimental results

\section{Quantal calculations}

3. Open-shell systems

Experimental results

Classical calculations

4. Summary

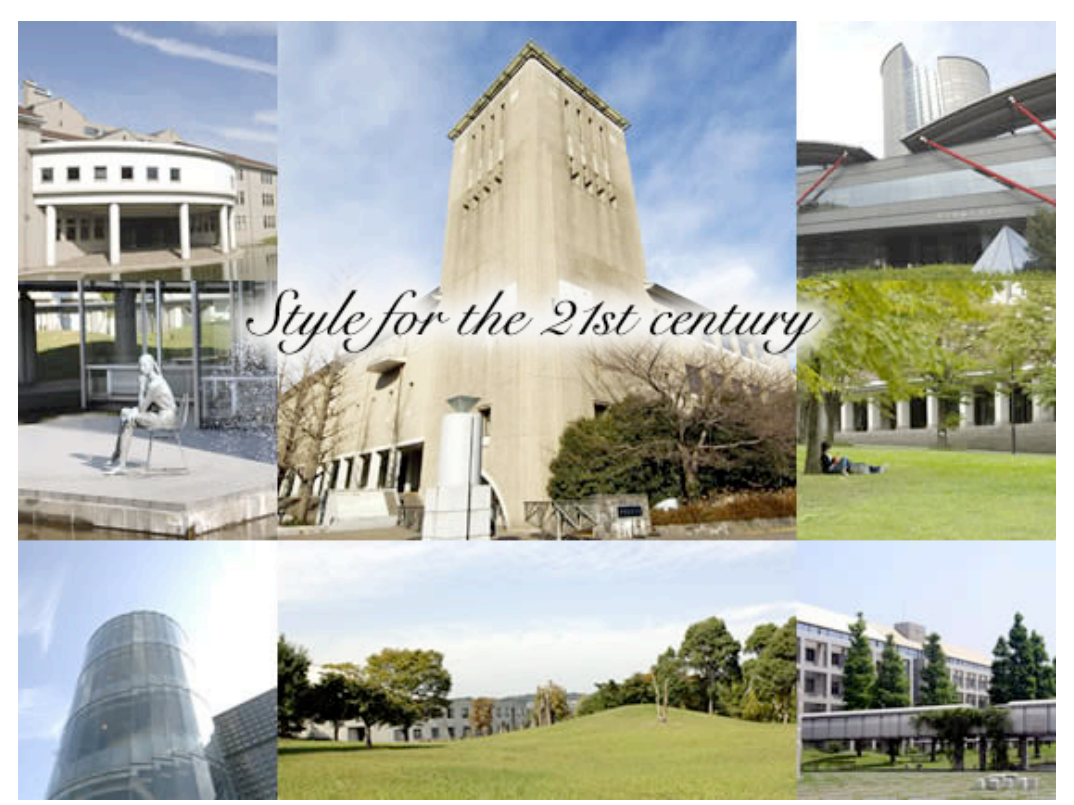




\section{Swarm \& Mobility}

\section{Swarm method :}

- long history since 19C

- extremely low energy collisions of ions / electrons

\section{Mobility :}

- fundamental transport property

- very sensitive to the interaction potential

- recent application to cluster ions - size and structure

- depend on electronic states

- historical works : $\mathrm{O}^{+}$and $\mathrm{O}_{2}{ }^{+}$

- this work: $\quad \mathrm{C}^{+}$and $\mathrm{N}^{+}$ 


\section{Principle of Ion Swarm Experiments}

\section{MS-DT-MS configuration}

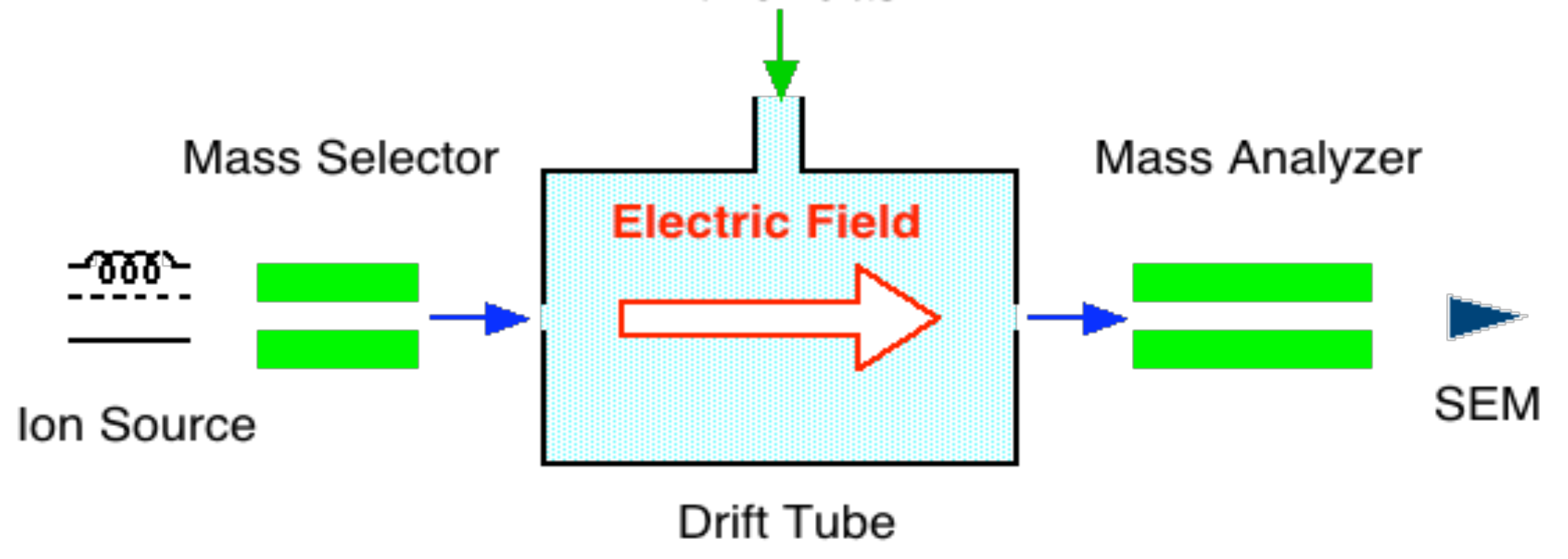

Experimental parameters which determine the collision energy

$$
\begin{array}{ll}
P & : \text { gas pressure }(N: \text { number density }) \\
E & : \text { uniform electric field strength } \\
T & : \text { gas temperature }
\end{array}
$$




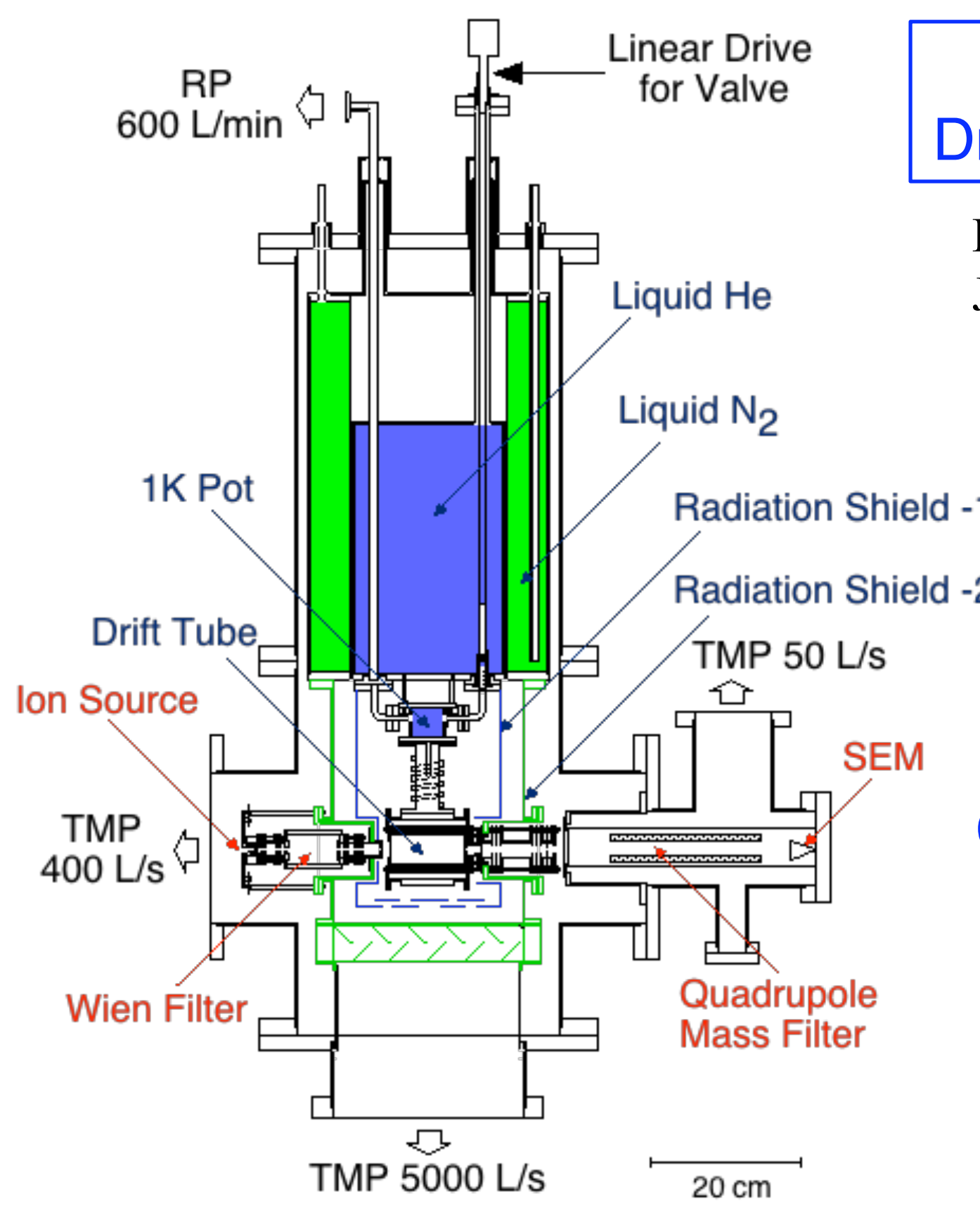

\section{Very Low Temperature} Drift Tube Mass Spectrometer

Rev. Sci. Instrum. 71 (2000) 2019.

J. Chem. Phys. 113 (2000) 1738.

$$
\begin{aligned}
& P=1-100 \mathrm{~Pa} \\
& E=0.1-20 \mathrm{~V} / \mathrm{cm} \\
& T=2.0-100 \mathrm{~K}
\end{aligned}
$$

Cooling by liq. $\mathrm{N}_{2}$ and liq. $\mathrm{He}$

Mean collision energy

$0.5<\varepsilon / \mathrm{meV}<1000$ 


\section{Drift velocity and Mobility of lons}

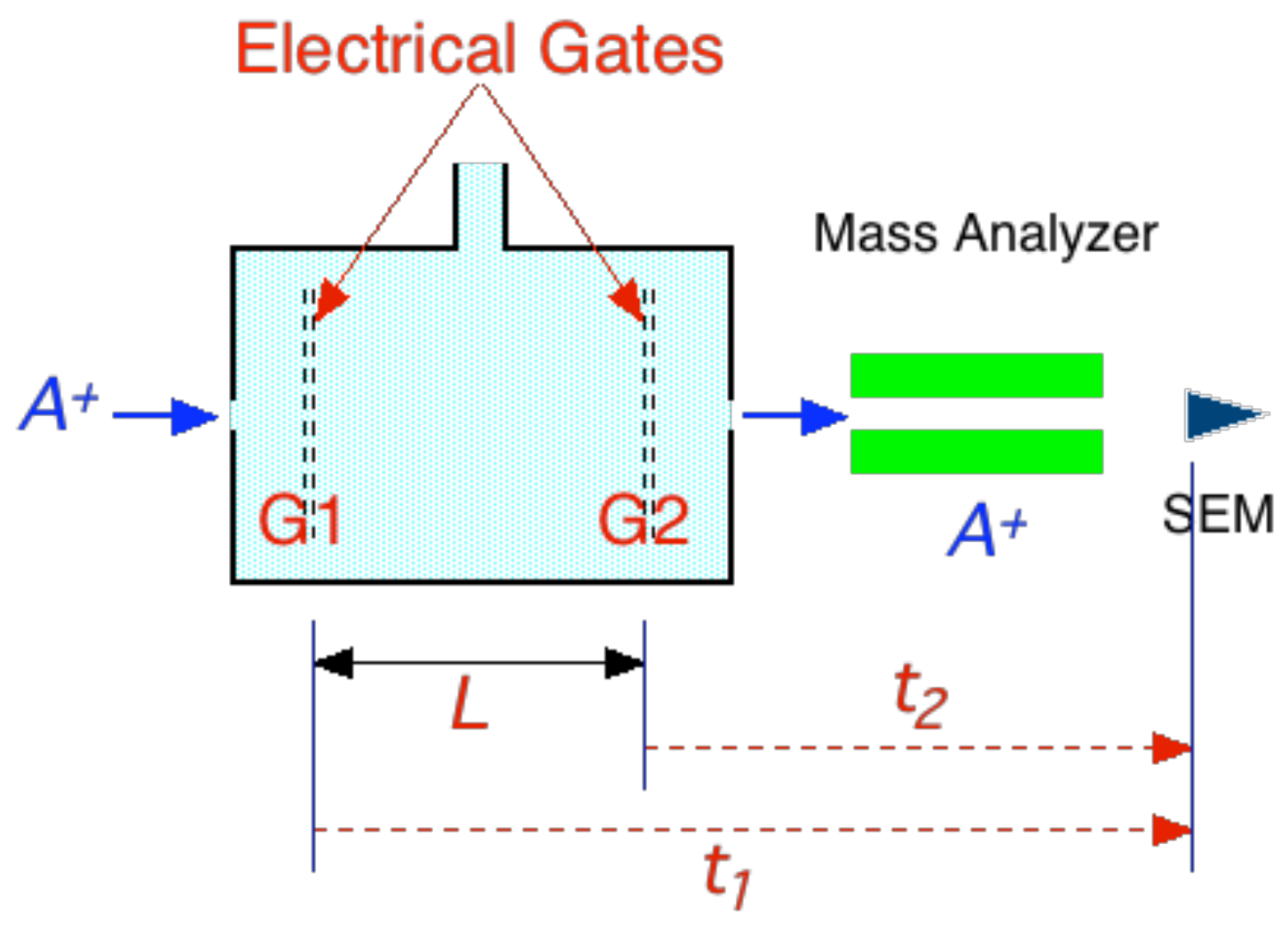

\section{Drift velocity :}

$$
v_{\mathrm{d}}=\frac{L}{t_{1}-t_{2}}=K E
$$

\section{Reduced mobility :}

$$
K_{0}=\frac{v_{\mathrm{d}}}{E} \frac{N}{N_{0}}=K_{0}\left(\frac{E}{N}\right)
$$

5 


\section{Typical Arrival Time Spectrum}

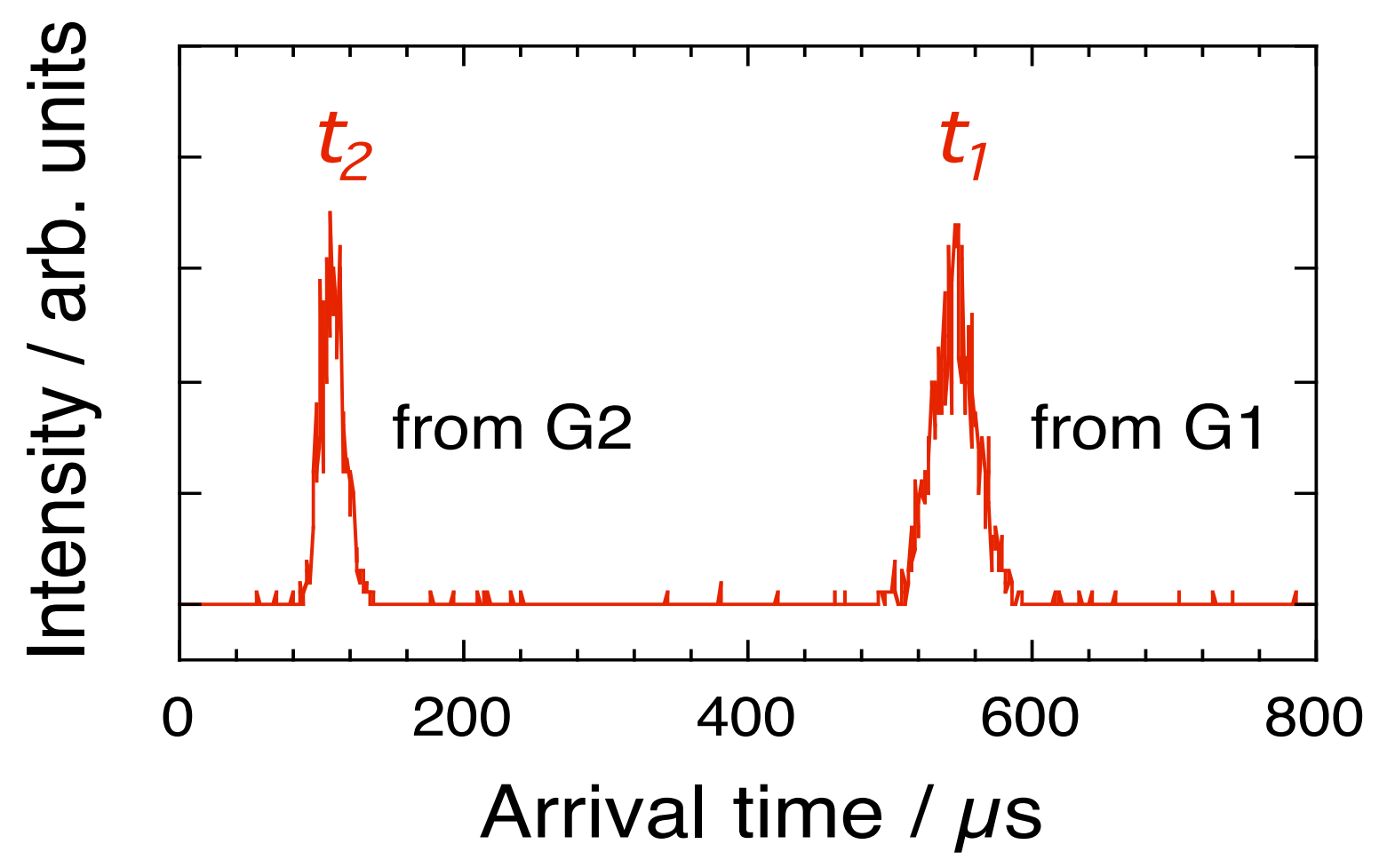

$\mathrm{He}^{+}$in $\mathrm{He}, P=2.1 \mathrm{~Pa}, E=0.5 \mathrm{~V} / \mathrm{cm}, T=4.3 \mathrm{~K}$ $v_{\mathrm{d}}=214 \mathrm{~m} / \mathrm{s}, K_{0}=24.4 \mathrm{~cm}^{2} \mathrm{~V}^{-1} \mathrm{~s}^{-1}$ 


\section{Scaling of mobility (1)}

reduced mobility

$$
K_{0}=\frac{v_{\mathrm{d}}}{E} \frac{N}{N_{0}}=K_{0}(T, E / N)
$$

$K_{0}$ generally does not depend on $P$.

mean collision energy $\quad\langle\varepsilon\rangle=\frac{3}{2} k T+\frac{1}{2} M v_{\mathrm{d}}^{2}=\frac{3}{2} k T_{\text {eff }}$

effective temperature $\quad T_{\text {eff }}=T+\frac{1}{3 k} M v_{\mathrm{d}}^{2}$

reduced mobility

$$
K_{0} \approx K_{0}\left(T_{\text {eff }}\right)
$$




\section{Scaling of mobility (2)}

polarization potential

$$
V_{\mathrm{pol}}(r)=-\frac{1}{4 \pi \varepsilon_{0}} \cdot \frac{e^{2} \alpha_{\mathrm{d}}}{2 r^{4}}
$$

polarization limit

$$
\begin{aligned}
K_{\mathrm{pol}} & \equiv K_{0}(E / N \rightarrow 0, T \rightarrow 0) \\
& =\frac{13.853}{\sqrt{\alpha_{\mathrm{d}} \mu}} \mathrm{cm}^{2} \mathrm{~V}^{-1} \mathrm{~s}^{-1}
\end{aligned}
$$

$\alpha_{\mathrm{d}}$ : polarizability of gas in $\AA^{3}$

$\mu$ : reduced mass in amu

$$
\text { normalized mobility }{ }^{\circledR} \quad \frac{K_{0}}{K_{\text {pol }}} \rightarrow 1 \begin{aligned}
& \left(T_{\text {eff }} \rightarrow 0 \mathrm{~K}\right) \\
& \text { in classical theory }
\end{aligned}
$$




\section{Compilations of experimental data}

E. A. Mason and his collaborators,

Transport properties of gaseous ions over a wide energy range Atomic Data and Nuclear Data Tables,

$\begin{array}{ll}\text { I } & \underline{17}, 177-210(1976), \\ \text { II } & \underline{22}, 179-217(1978), \\ \text { III } & \underline{31}, 113-151(1984), \\ \text { IV } & \underline{60}, 37-95(1995) .\end{array}$




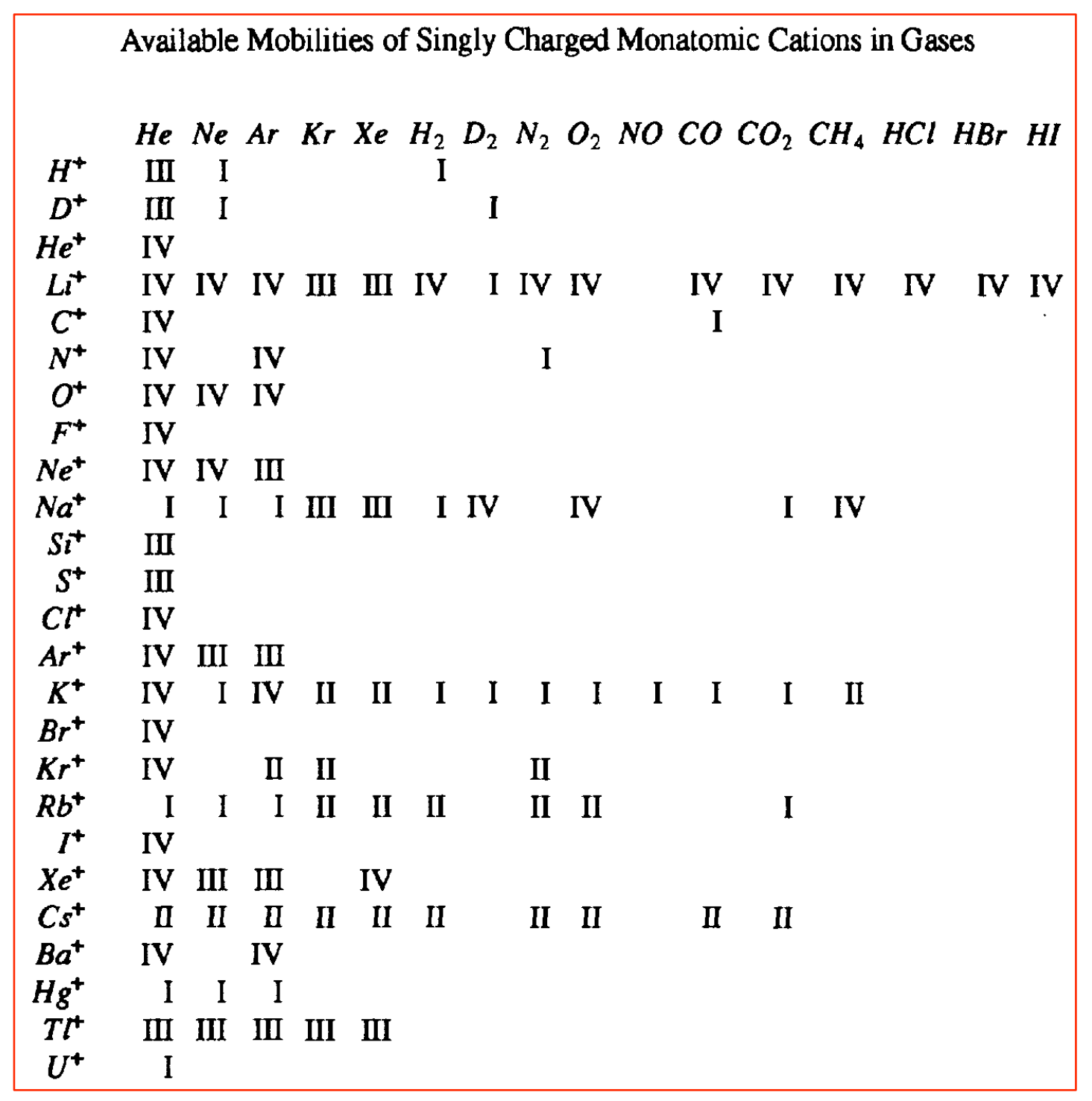


Available Mobilities of Singly Charged Polyatomic Cations in Gases

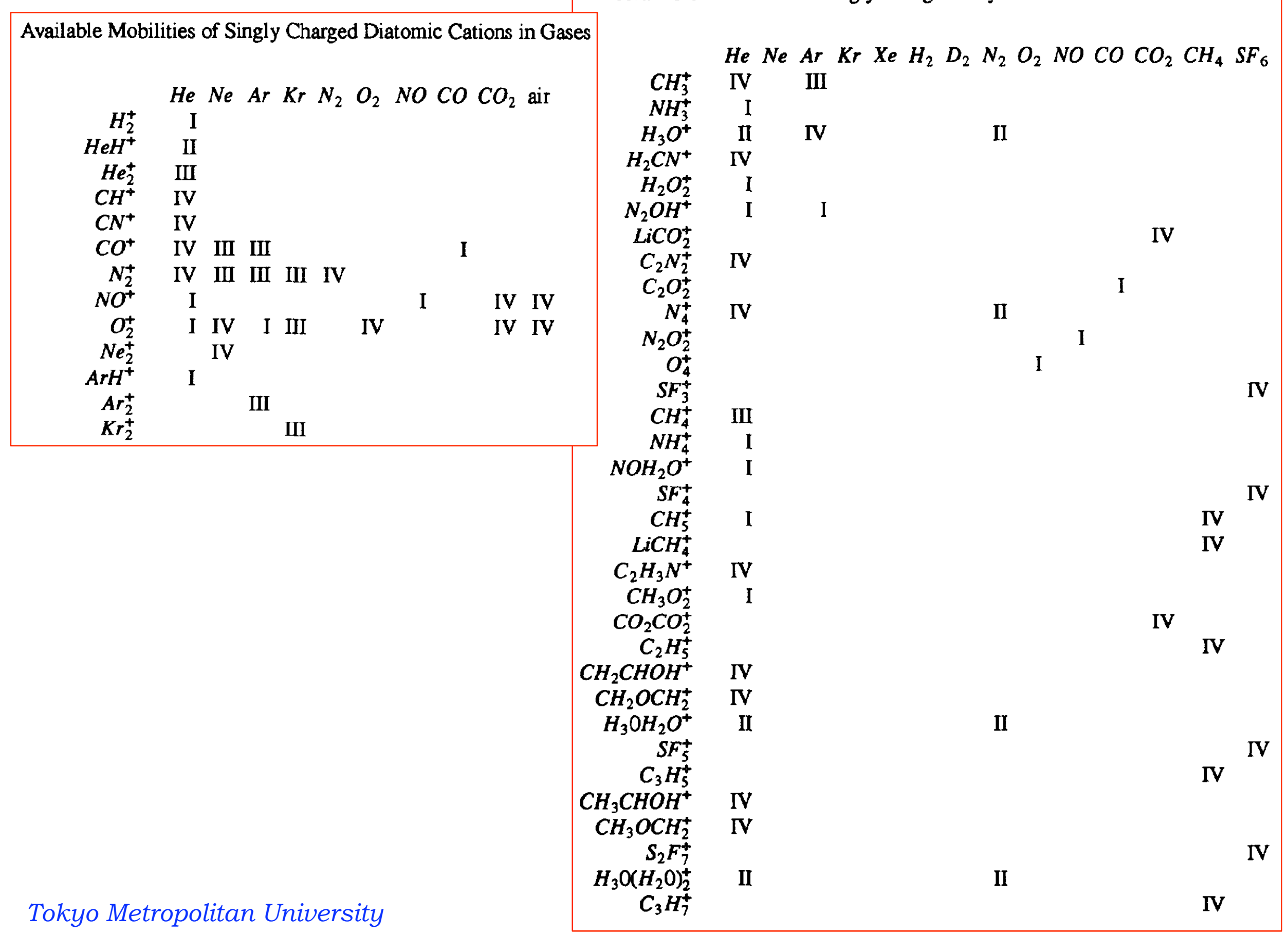




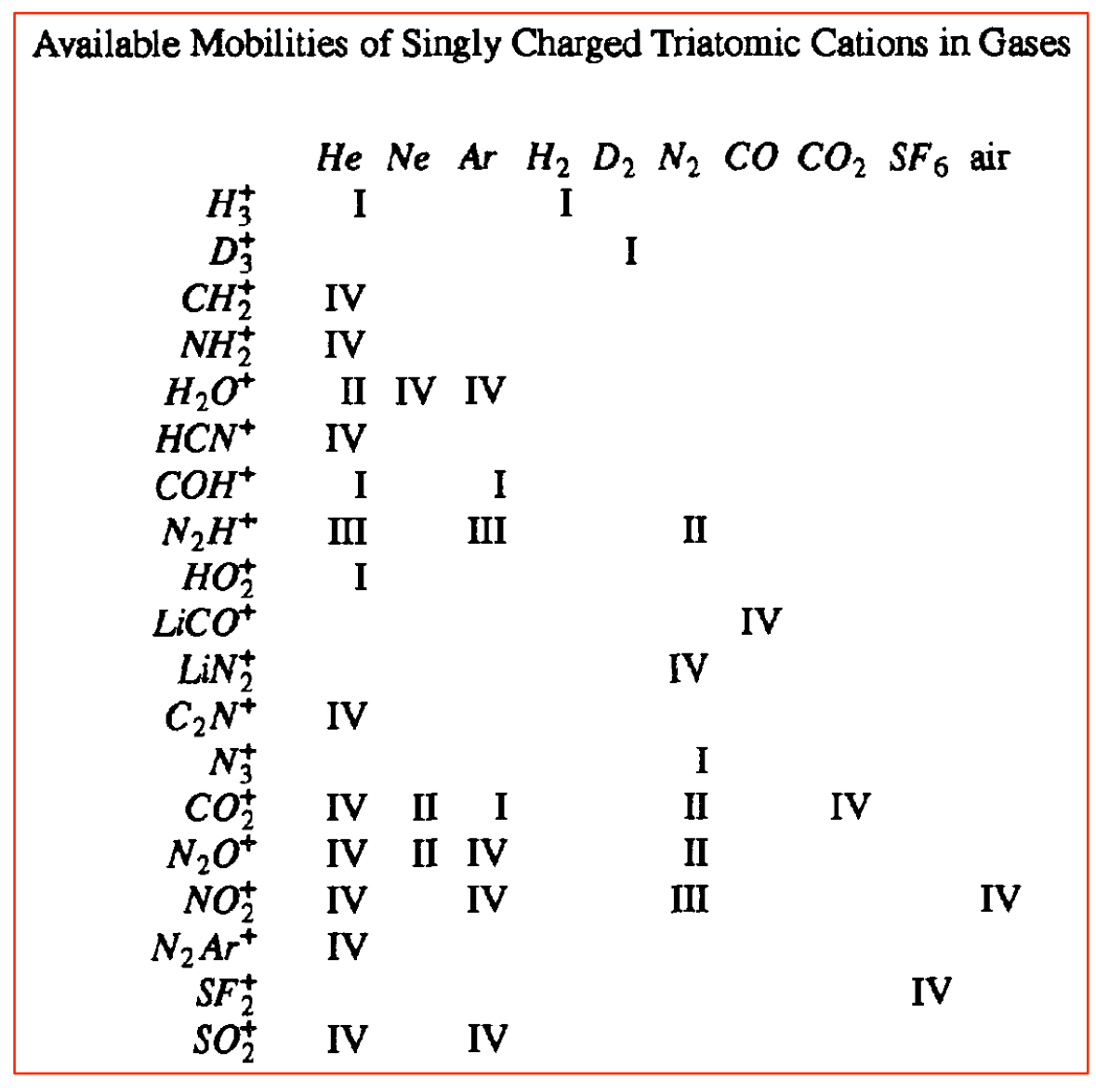

Available Mobilities of Doubly Charged Cations in Gases

He Ne Ar $\mathrm{Kr} \mathrm{Xe}$

$$
\begin{array}{ccccccc}
H e^{2+} & \text { II } & & & & \\
N e^{2+} & \text { III } & \text { II } & & & \\
A r^{2+} & \text { III } & & \text { II } & & \\
K r^{2+} & \text { IV } & & & \text { IV } & \\
X e^{2+} & \text { IV } & \text { III } & & & \text { II } \\
O^{2+} & \text { IV } & \text { IV } & & & & \\
H g^{2+} & \text { IV } & & & & \\
O_{2}^{2+} & \text { IV } & & \text { IV } & & &
\end{array}
$$

\begin{tabular}{|c|c|c|c|c|c|c|c|c|c|}
\hline \multicolumn{10}{|c|}{ Available Mobilities of Singly Charged Anions in Gases } \\
\hline & He & $\mathrm{Ne}$ & $A r K r$ & $X e$ & $\mathrm{H}_{2} \mathrm{~N}_{2}$ & $O_{2}$ & $\mathrm{CO}_{2}$ & $S F_{6}$ & air \\
\hline$H^{-}$ & I & & & & & & & & \\
\hline$O^{-}$ & IV & & & & & I & I & & IV \\
\hline$F^{-}$ & IV & & II II & II & & & & IV & \\
\hline$S^{-}$ & IV & & & & & & & & \\
\hline $\mathrm{Cl}^{-}$ & IV & II & II II & II & III & & & & IV \\
\hline $\mathrm{Br}^{-}$ & II & III & III III & III & & & & & \\
\hline$I^{-}$ & II & & II & & & & & & \\
\hline$O H^{-}$ & IV & & & & & & & & \\
\hline$O D^{-}$ & IV & & & & & & & & \\
\hline $\mathrm{O}_{2}^{-}$ & IV & & & & & I & & & IV \\
\hline $\mathrm{SH}^{-}$ & IV & & & & & & & & \\
\hline$S_{2}^{-}$ & IV & & & & & & & & \\
\hline $\mathrm{NO}_{2}^{-}$ & I & & & & III & & & & IV \\
\hline$O_{3}^{-}$ & I & & I & & & I & & & IV \\
\hline $\mathrm{C}_{2} \mathrm{H}_{2}^{-}$ & I & & & & & & & & \\
\hline $\mathrm{CO}_{3}^{-}$ & I & & I & & & I & $I$ & & \\
\hline $\mathrm{NO}_{3}^{-}$ & & & & & III & & & & \\
\hline $\mathrm{SO}_{2} \mathrm{~F}^{-}$ & I & & & & & & & & \\
\hline $\mathrm{SO}_{3}^{-}$ & I & & & & & & & & \\
\hline $\mathrm{CO}_{4}^{-}$ & & & & & & I & & & \\
\hline$S F_{5}^{-}$ & I & & & & & & & IV & \\
\hline$S F_{6}^{-}$ & I & & & & & & & IV & \\
\hline$S F_{6} S F_{6}^{-}$ & & & & & & & & IV & \\
\hline$S F_{6}\left(S F_{6}\right)_{2}$ & & & & & & & & IV & \\
\hline
\end{tabular}




\section{closed-shell systems}




\section{Our works - Ion mobility in cooled He gas}

Atomic lons :

$$
\begin{aligned}
& \mathrm{H}^{+} \\
& \mathrm{He}^{+}, \mathrm{Ne}^{+}, \mathrm{Ar}^{+}, \mathrm{Kr}^{+}, \mathrm{Xe}^{+} \\
& \mathrm{Li}^{+}, \mathrm{Na}^{+}, \mathrm{K}^{+}, \mathrm{Rb}^{+}, \mathrm{Cs}^{+} \\
& \mathrm{C}^{+}, \mathrm{N}^{+}, \mathrm{O}^{+}
\end{aligned}
$$

Molecular Ions :

$$
\begin{aligned}
& \mathrm{N}_{2}^{+}, \mathrm{O}_{2}^{+}, \mathrm{CO}^{+}, \mathrm{NO}^{+}, \mathrm{CH}^{+} \\
& \mathrm{CO}_{2}{ }^{+}, \mathrm{NO}_{2}{ }^{+}, \mathrm{CH}_{2}{ }^{+} \\
& \mathrm{CH}_{3}{ }^{+} \\
& \mathrm{CO}_{2}{ }^{2+}
\end{aligned}
$$




\section{Mobility of $\mathrm{Li}^{+}$in $\mathrm{He}$ at 4.3, 77, and $300 \mathrm{~K}$}
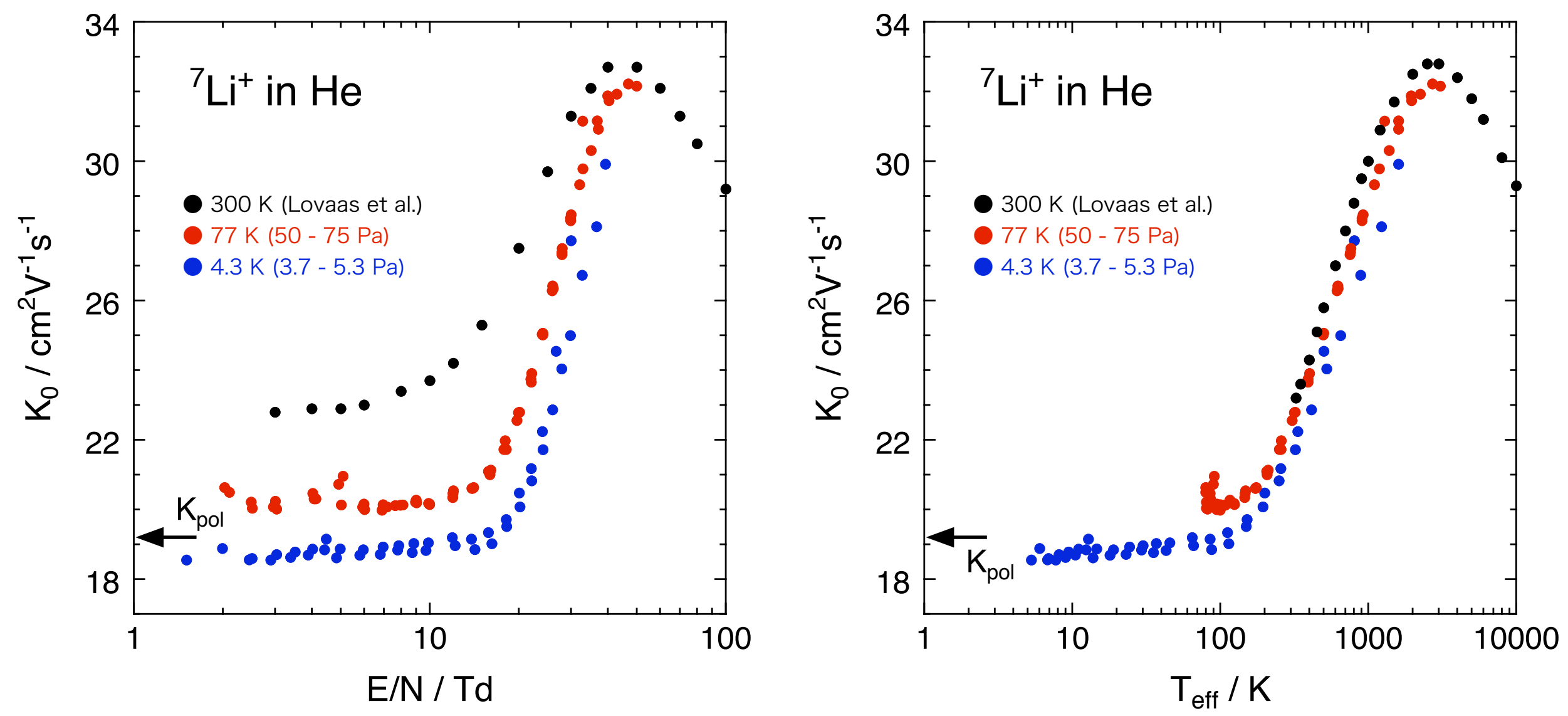

Scaling by $T_{\text {eff }}$ is sufficient to compile the mobility data measured at different gas temperatures. 


\section{Two-temperature Theory of Ion Mobility}

Mobility

$$
K_{0}=\frac{3 e}{16 N_{0}}\left(\frac{2 \pi}{\mu k T_{\text {eff }}}\right)^{1 / 2} \frac{1+\alpha}{\Omega^{(1,1)}\left(T_{\text {eff }}\right)}
$$

Effective temp.

$$
T_{\text {eff }}=T+\frac{1}{3 k} M v_{\mathrm{d}}^{2}(1+\beta)
$$

Collision integral

$$
\Omega^{(1,1)}\left(T_{\text {eff }}\right)=\frac{1}{2\left(k T_{\text {eff }}\right)^{3}} \int_{0}^{\infty} Q^{(1)}(\varepsilon) \exp \left(-\frac{\varepsilon}{k T_{\text {eff }}}\right) \varepsilon^{2} \mathrm{~d} \varepsilon
$$

Momentum transfer cross section

$$
\begin{array}{r}
Q^{(1)}(\varepsilon)=\frac{4 \pi}{\kappa^{2}} \sum_{l=0}^{\infty}(l+1) \sin ^{2}\left(\eta_{l}-\eta_{l+1}\right) \\
\kappa=\sqrt{2 \mu \varepsilon} / \hbar \quad \eta_{l}: \text { phase shift }
\end{array}
$$




\section{Potential curve and MTCS of $\mathrm{Li}^{+}$in $\mathrm{He}$}
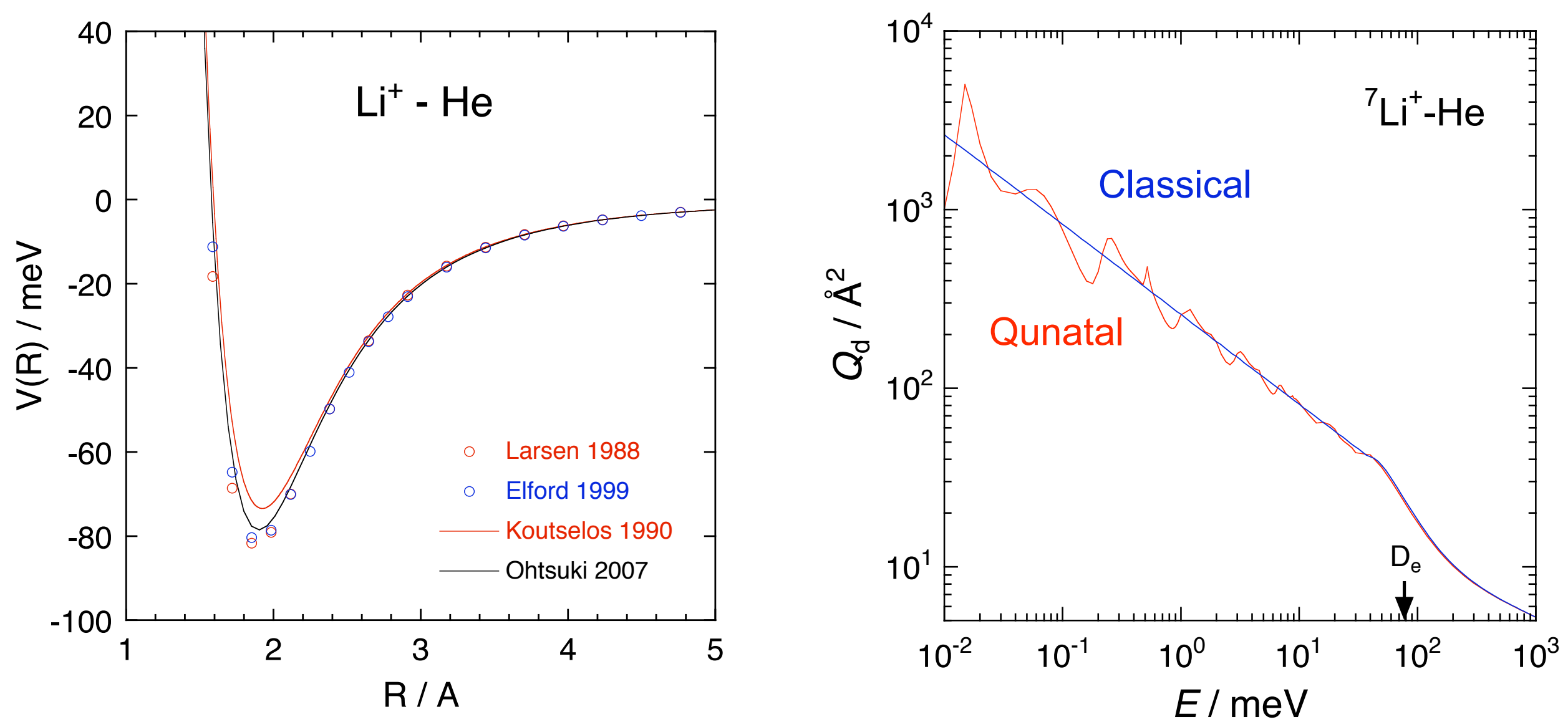


\section{Potential curve and Mobility of $\mathrm{Li}^{+}$in $\mathrm{He}$}
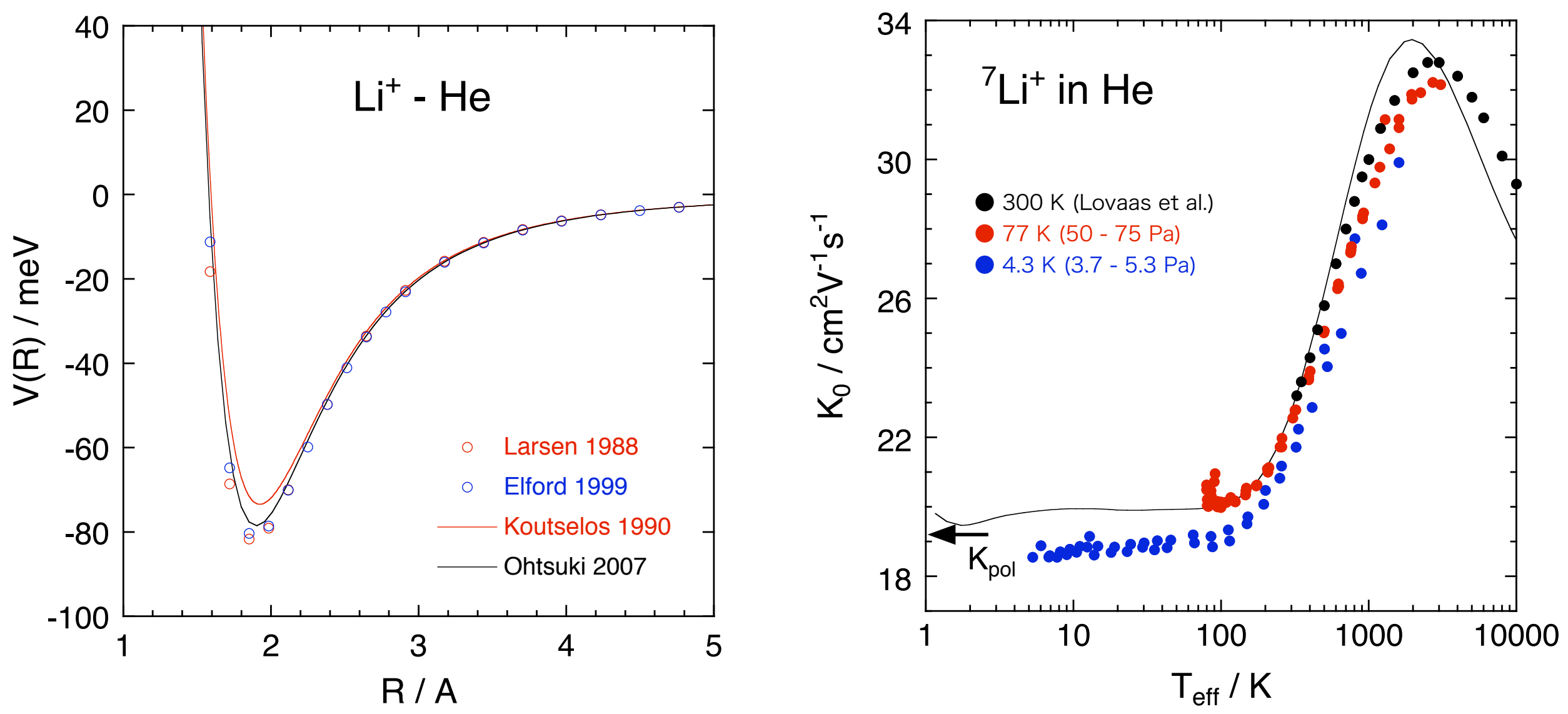

Accurate potential gives good agreement between the experiment value and the theoretical calculation. 


\section{$K_{0} / K_{\mathrm{pol}}$ of five alkali ions in He}
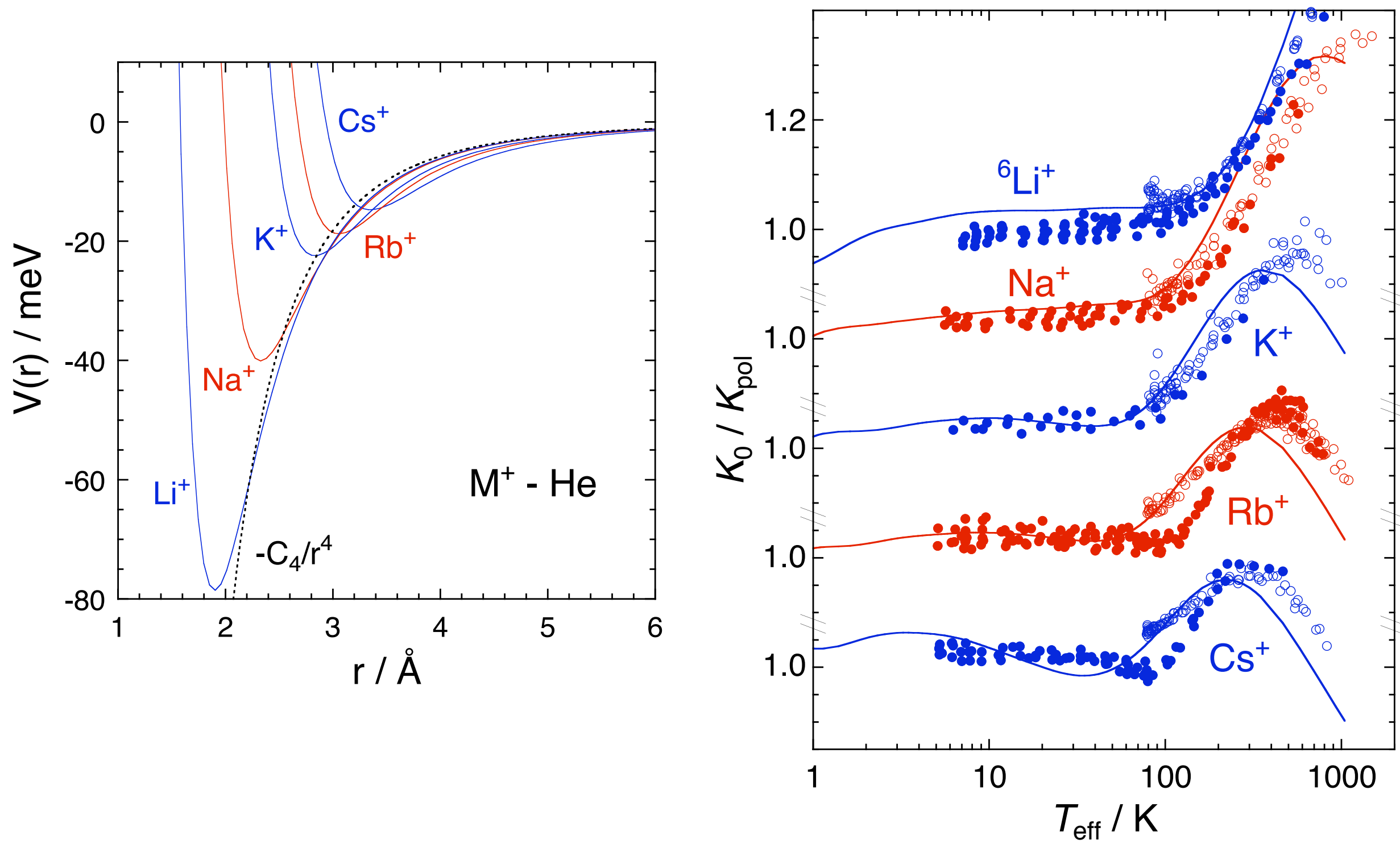


\section{Historical works in Georgia Institute of Technology @ room temperature}

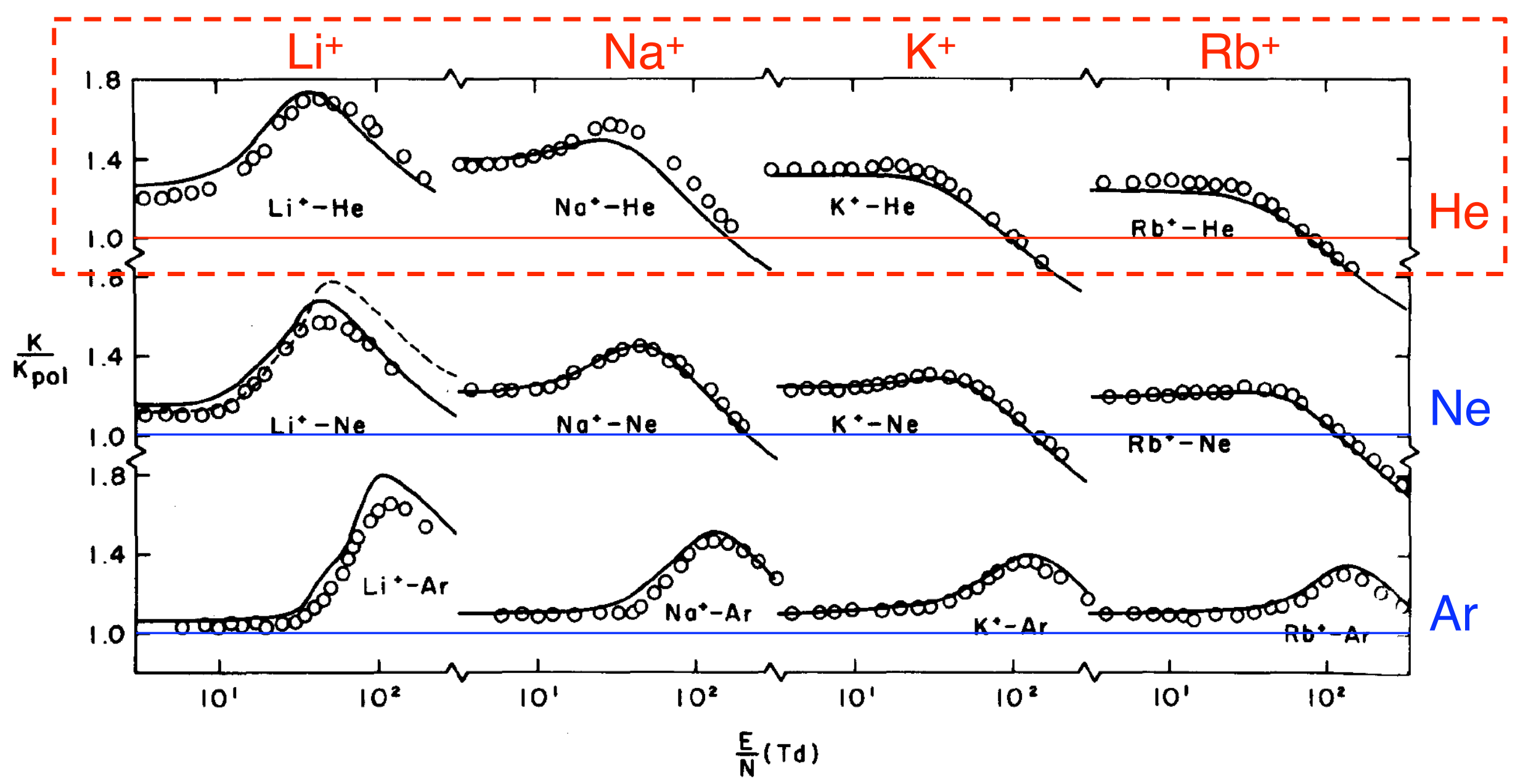

I. R. Gatland et al., J. Chem. Phys. $\underline{66}$ (1977) 537. 


\section{Generalized ion mobility curve}

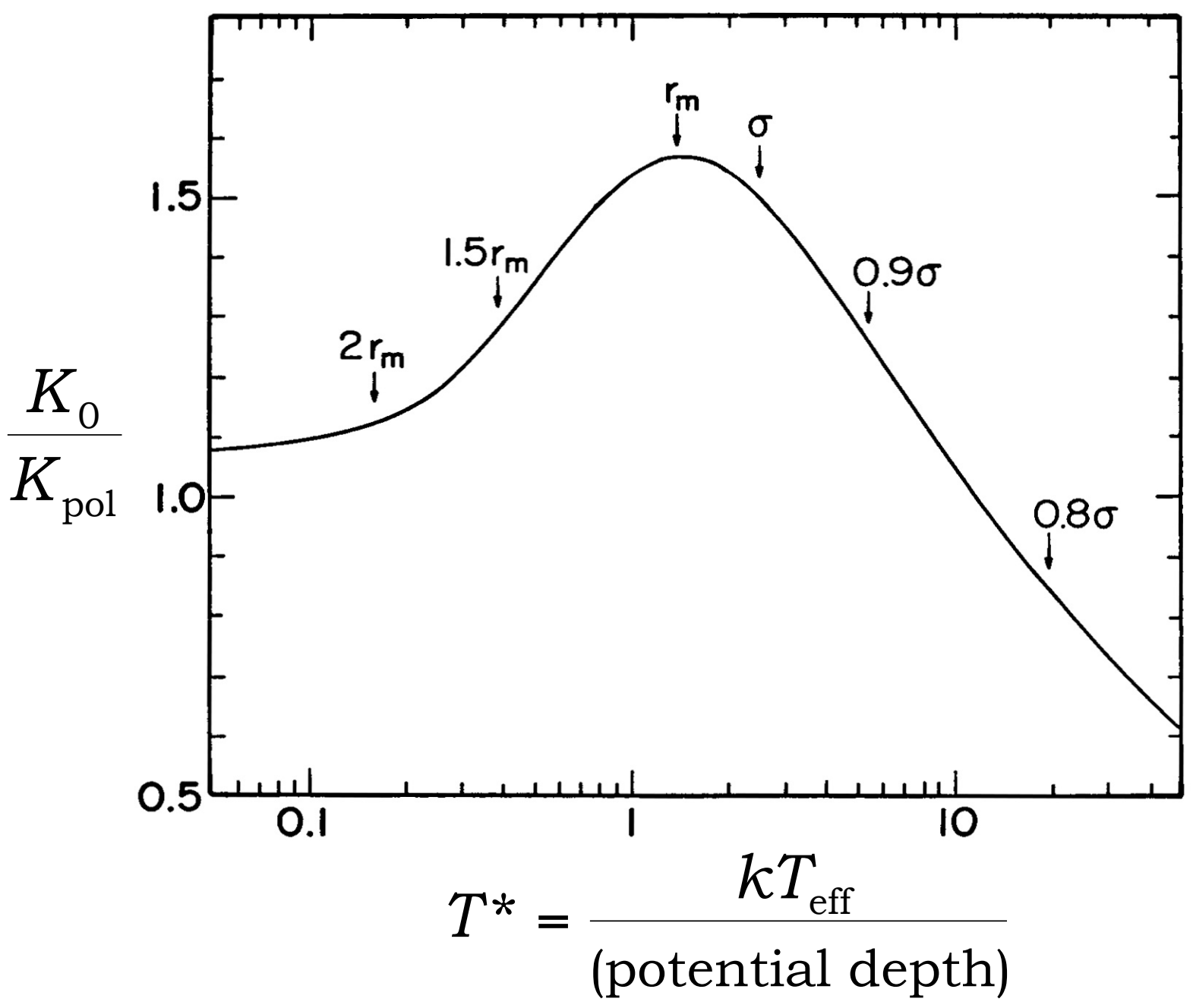




\section{open-shell systems}




\section{Meta-stable States of Atomic Ions}

\section{Ion Configuration Energy Fraction* Source gas}

$\begin{array}{lllrl}\mathrm{C}^{+}\left({ }^{2} \mathrm{P} \circ\right. & 2 \mathrm{~s}^{2} 2 \mathrm{p} & \text { G.S. } & 94 \% & \mathrm{CH}_{4} \\ \mathrm{C}^{+}\left({ }^{4} \mathrm{P}\right) & 2 \mathrm{~s} 2 \mathrm{p}^{2} & 5.3 \mathrm{eV} & 6 \% & \end{array}$

$\mathrm{N}^{+}\left({ }^{3} \mathrm{P}\right) \quad 2 \mathrm{~s}^{2} 2 \mathrm{p}^{2} \quad$ G.S. $\quad 88 \%$

$\mathrm{N}^{+}\left({ }^{1} \mathrm{D}\right) \quad 2 \mathrm{~s}^{2} 2 \mathrm{p}^{2} \quad 1.9 \mathrm{eV} \quad 12 \% \quad \mathrm{~N}_{2}$

$\begin{array}{llll}\mathrm{N}^{+}\left({ }^{1} \mathrm{~S}\right) & 2 \mathrm{~s}^{2} 2 \mathrm{p}^{2} & 4.1 \mathrm{eV} & - \\ \mathrm{O}^{+}\left({ }^{4} \mathrm{~S}^{\circ}\right) & 2 \mathrm{~s}^{2} 2 \mathrm{p}^{3} & \text { G.S. } & 65 \%\end{array}$

$\mathrm{O}^{+}\left({ }^{2} \mathrm{D}^{\circ}\right) \quad 2 \mathrm{~s}^{2} 2 \mathrm{p}^{3} \quad 3.3 \mathrm{eV} \quad 15 \%$

$\mathrm{O}^{+}\left({ }^{2} \mathrm{P}^{\circ}\right) \quad 2 \mathrm{~s}^{2} 2 \mathrm{p}^{3} \quad 5.0 \mathrm{eV} \quad 20 \%$

*electron impact of 70 eV, Enos et al., J. Phys B 25 (1992) 4021. 


\title{
Arrival spectra of $\mathrm{C}^{+}$in $\mathrm{He}$ at $4.3 \mathrm{~K}$
}

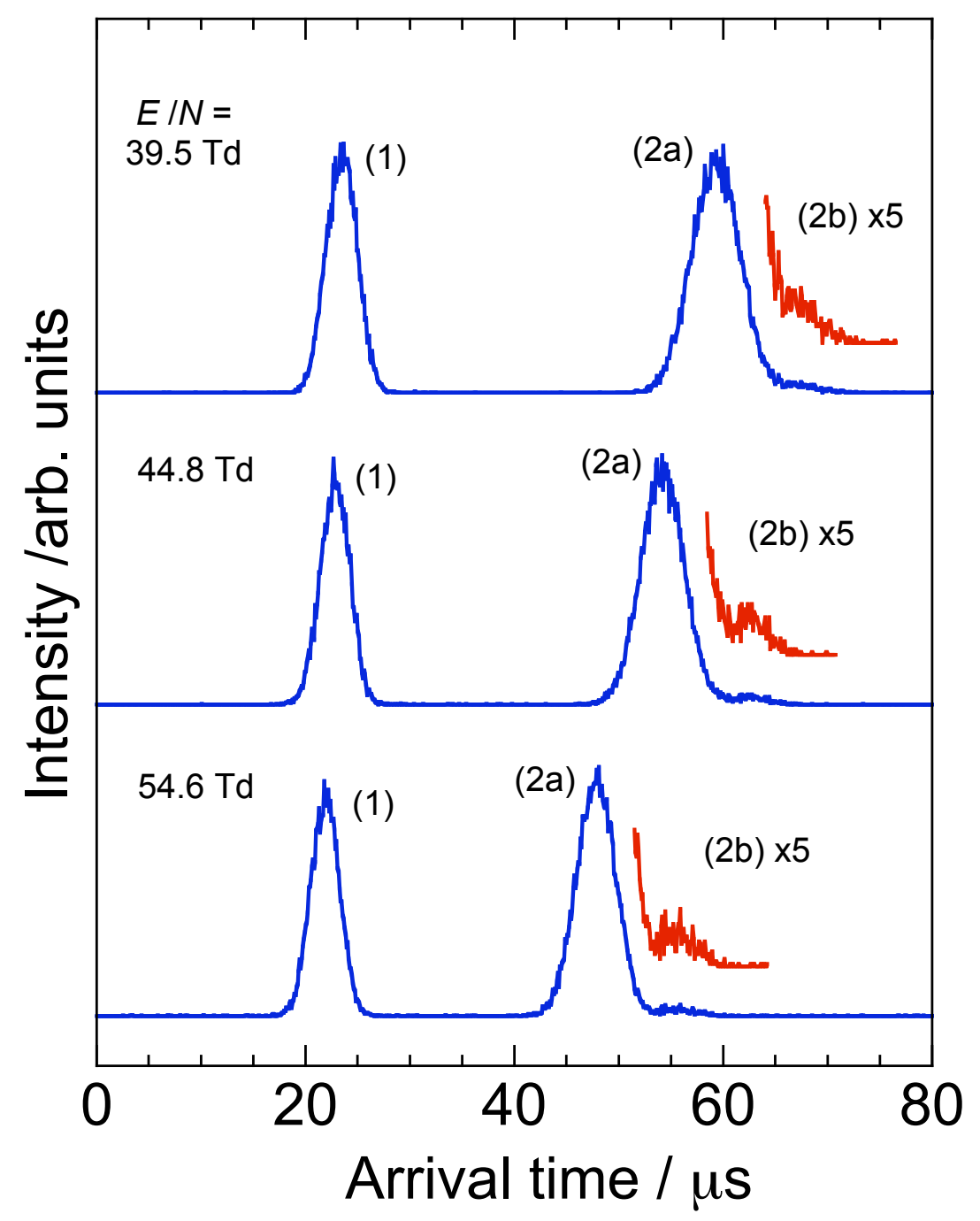

\author{
Fraction Identification \\ (2a) $98 \%$ G.S. ( $\left.{ }^{2} \mathrm{P}\right)$ \\ (2b) 2 - $3 \%$ M.S. ( $\left.{ }^{4} \mathrm{P}\right)$
}




\section{Mobility of $\mathrm{C}^{+}$in He at 4.3, 77, and $300 \mathrm{~K}$}
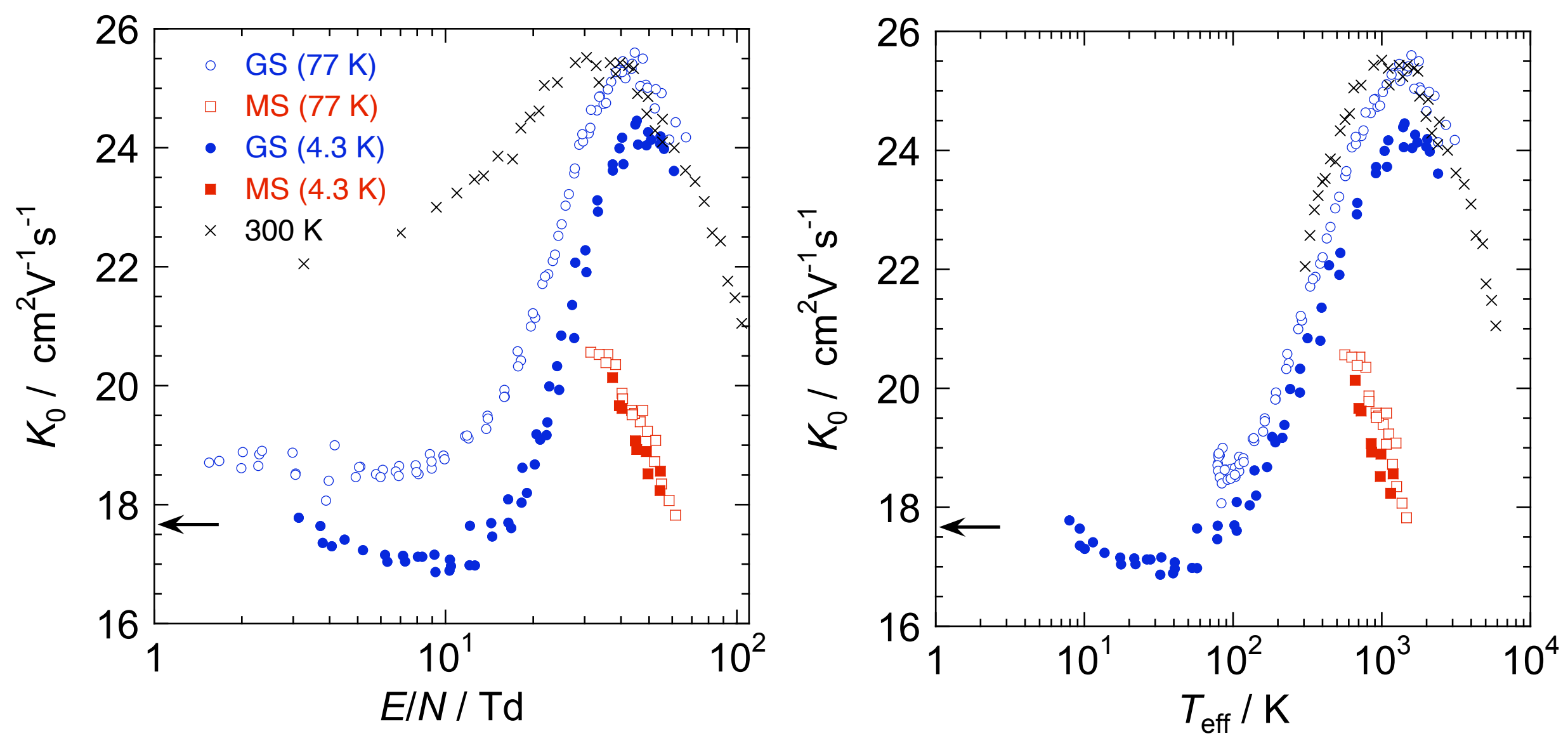


\section{ab initio potentials and cross sections}

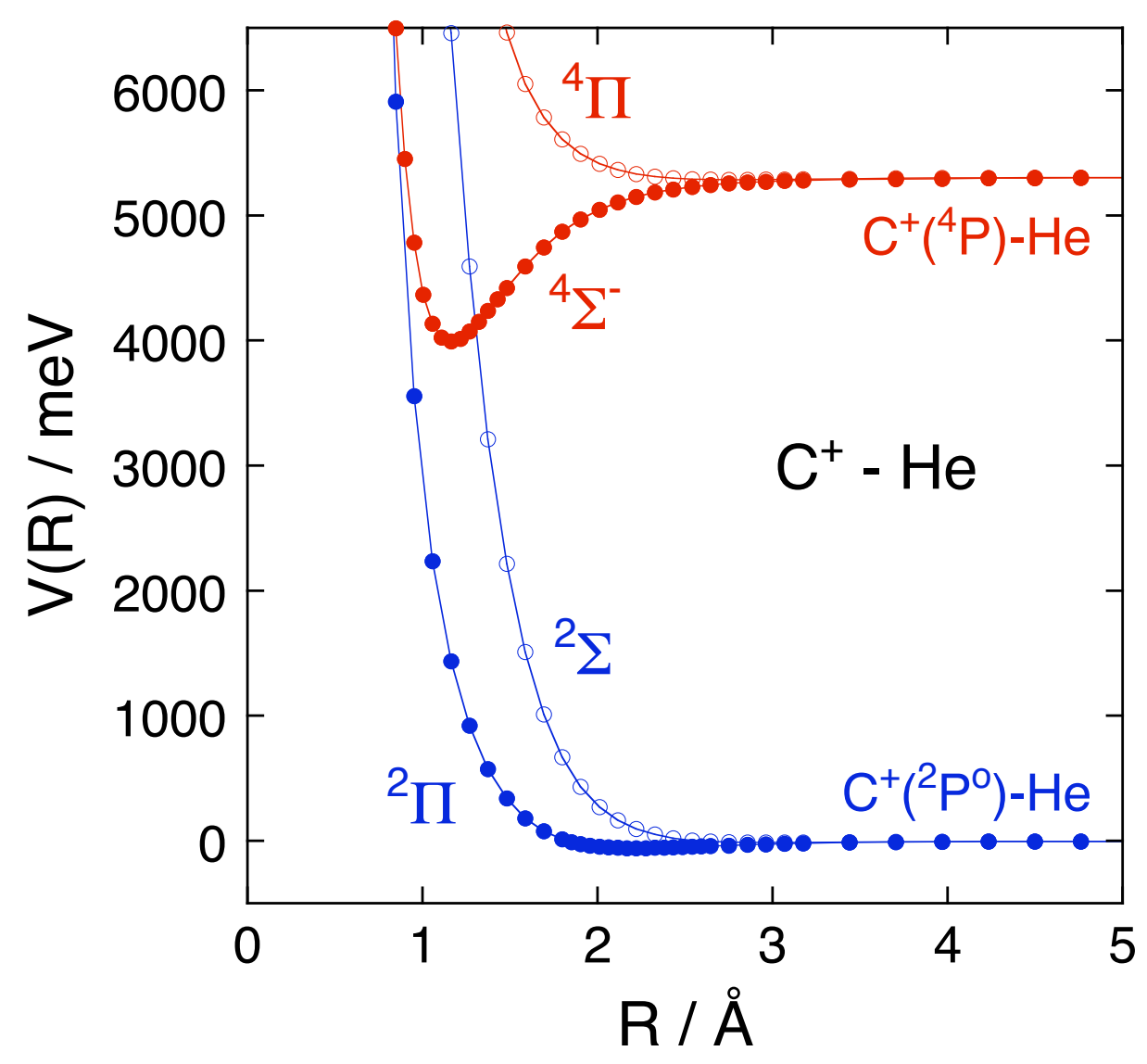

Approximated cross sections

$$
Q \approx \frac{g_{a} Q_{a}+g_{b} Q_{b}}{g_{a}+g_{b}}
$$

$g_{\mathrm{i}}:$ multiplicity of the state $i$

$$
\begin{array}{ll}
\Sigma: & g=1 \\
\Pi: & g=2 \\
\Delta: & g=2
\end{array}
$$

As $Q_{i}$, we used the classical cross sections. 


\section{Calculation for the ground state $\mathrm{C}^{+}\left({ }^{2} \mathrm{P}\right)$}
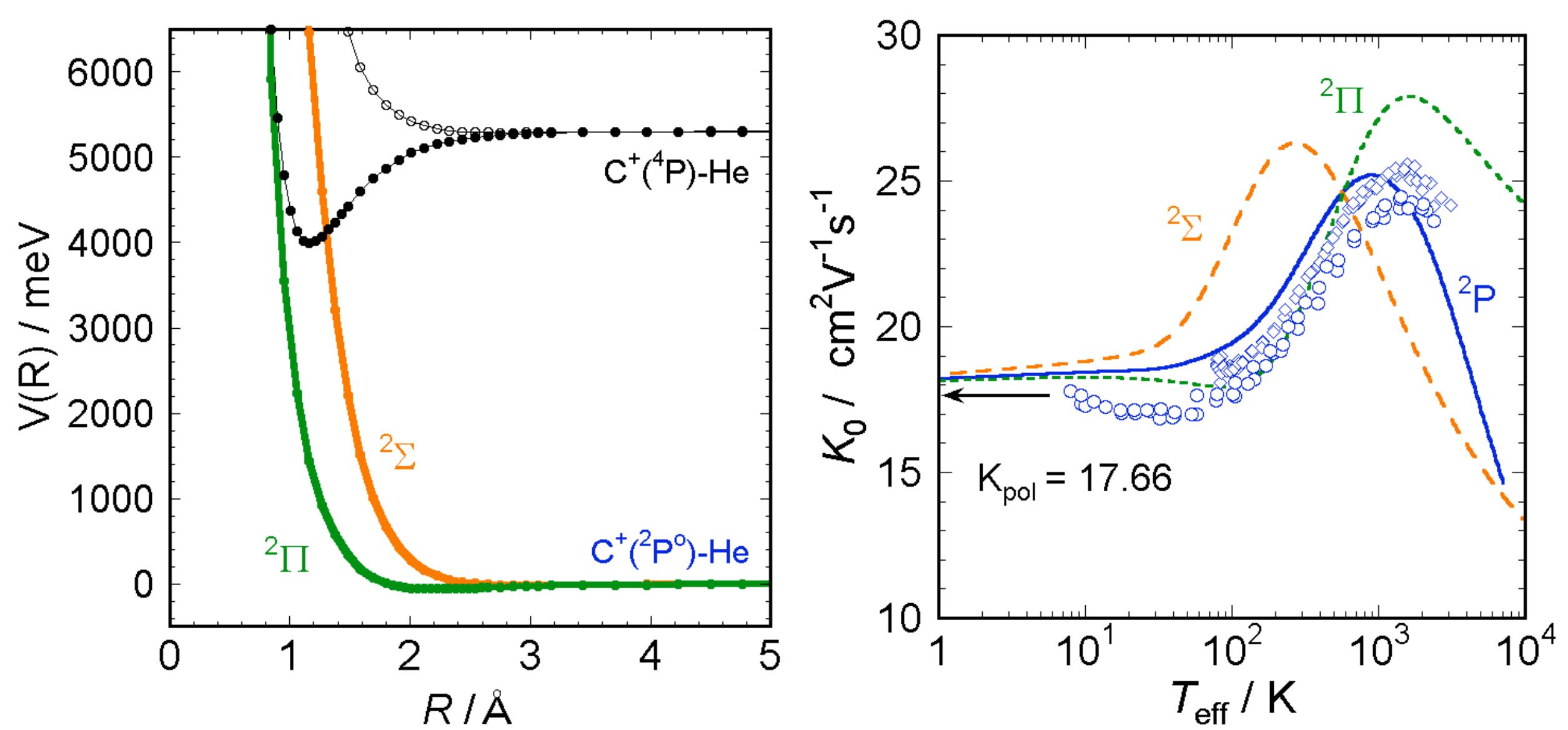

Calculation with only one potential can not reproduce the measured mobility. 


\section{Calculation for the matastable state $C^{+}\left({ }^{4} P\right)$}
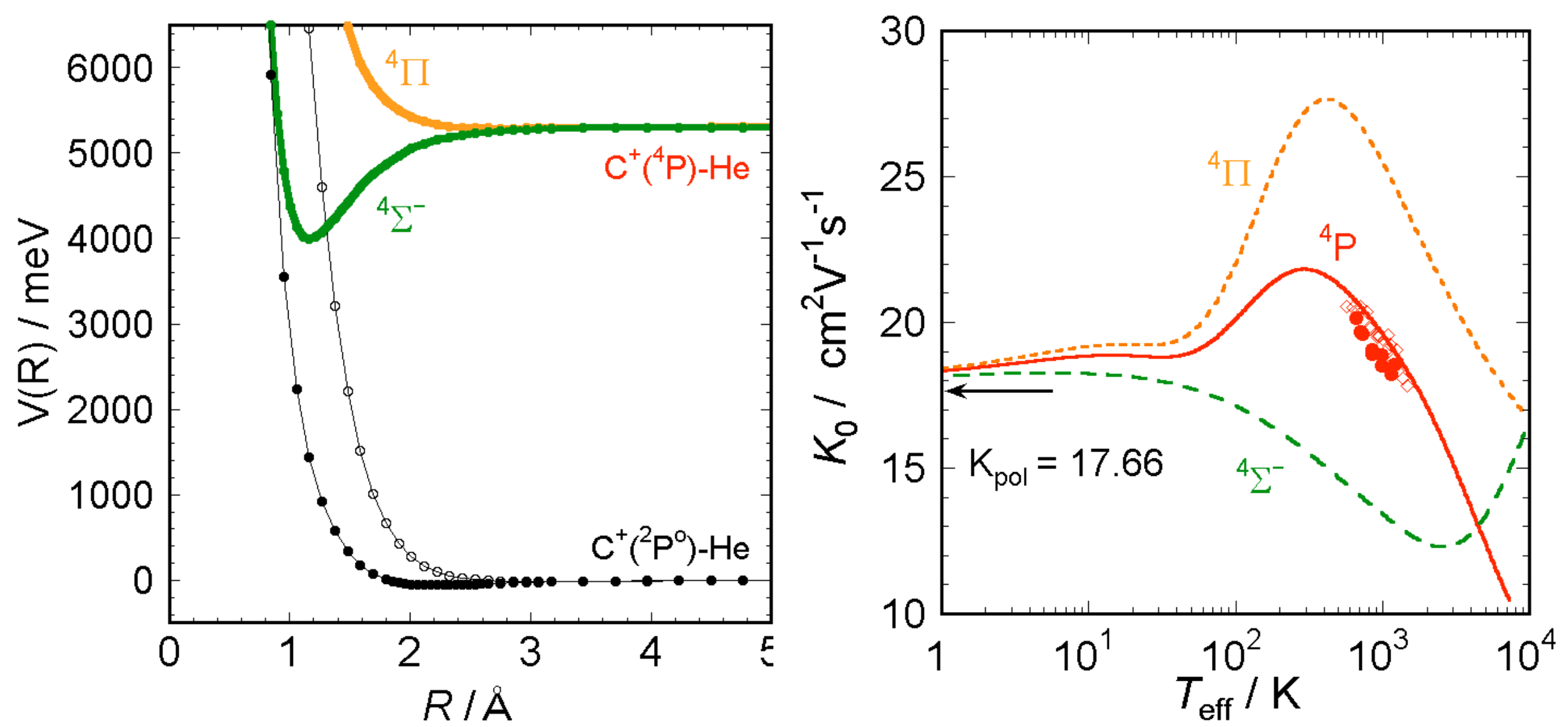

Calculation with two potentials reproduces the experiment even though the interaction between two states is neglected. 


\section{Mobility of $C\left({ }^{2} P\right)$ and $C^{+}\left({ }^{4} P\right)$ in He}

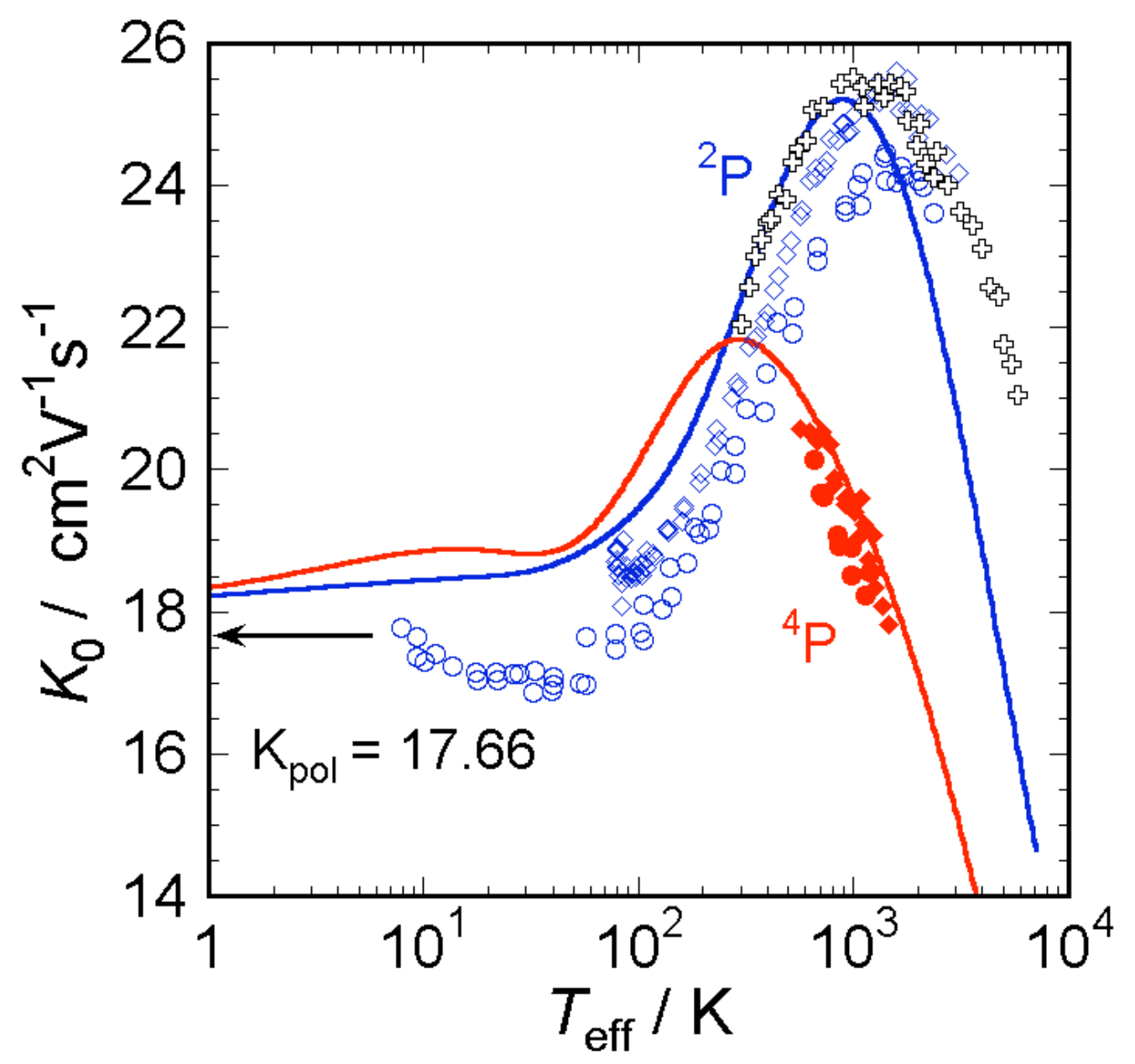


Arrival spectra of $\mathrm{N}^{+}$in $\mathrm{He}$ at $4.3 \mathrm{~K}$

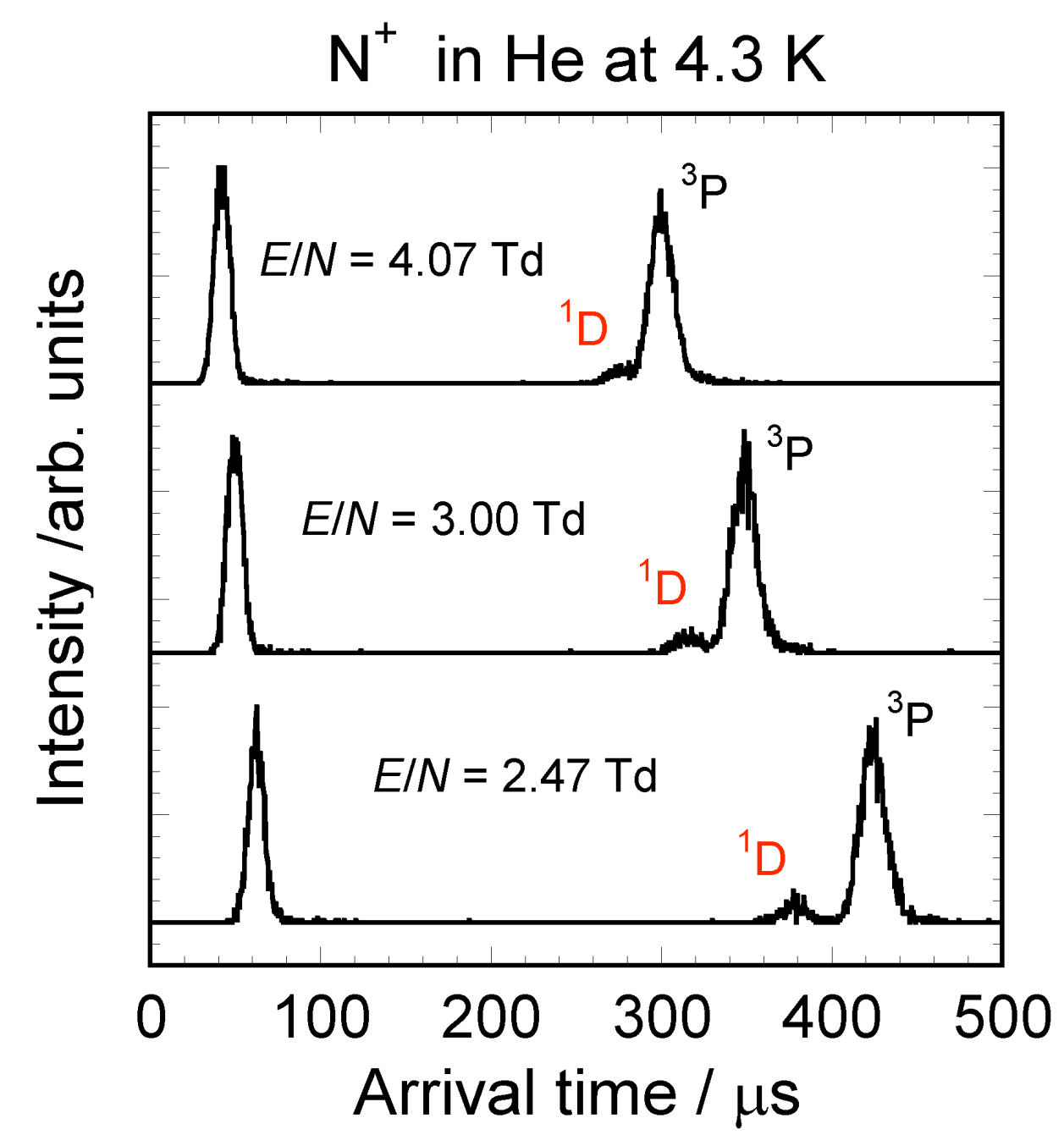

This work Eos et al.

G.S. ( $\left.{ }^{3} P\right) \quad 72-95 \% \quad 88 \%$

M.S. $\left({ }^{1} D\right) \quad 5-28 \% \quad 12 \%$

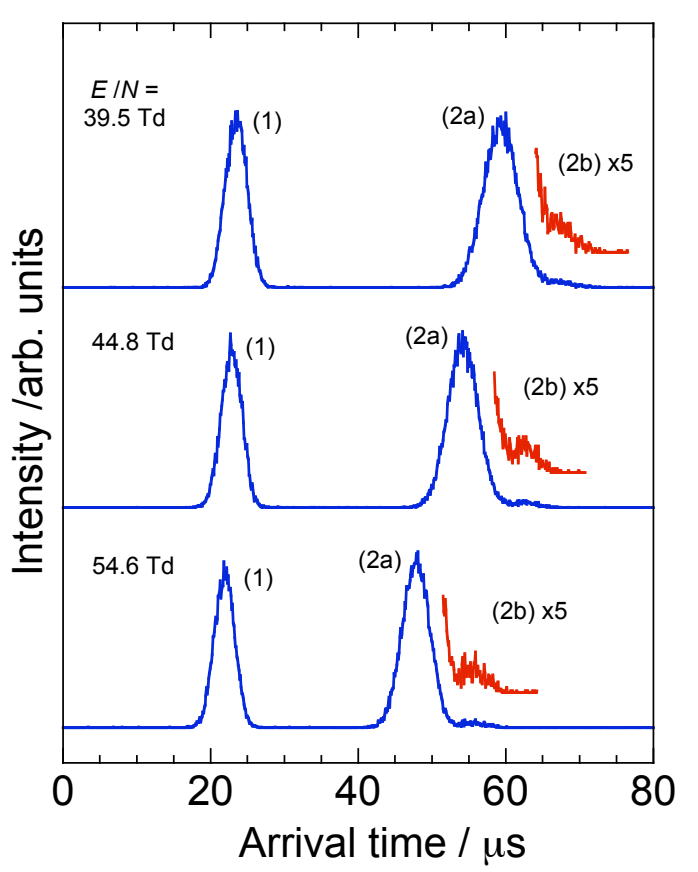

$\mathrm{C}^{+} / \mathrm{He}$ 


\section{Mobility of $\mathrm{N}^{+}$in $\mathrm{He}$ at $4.3,77$, and $300 \mathrm{~K}$}
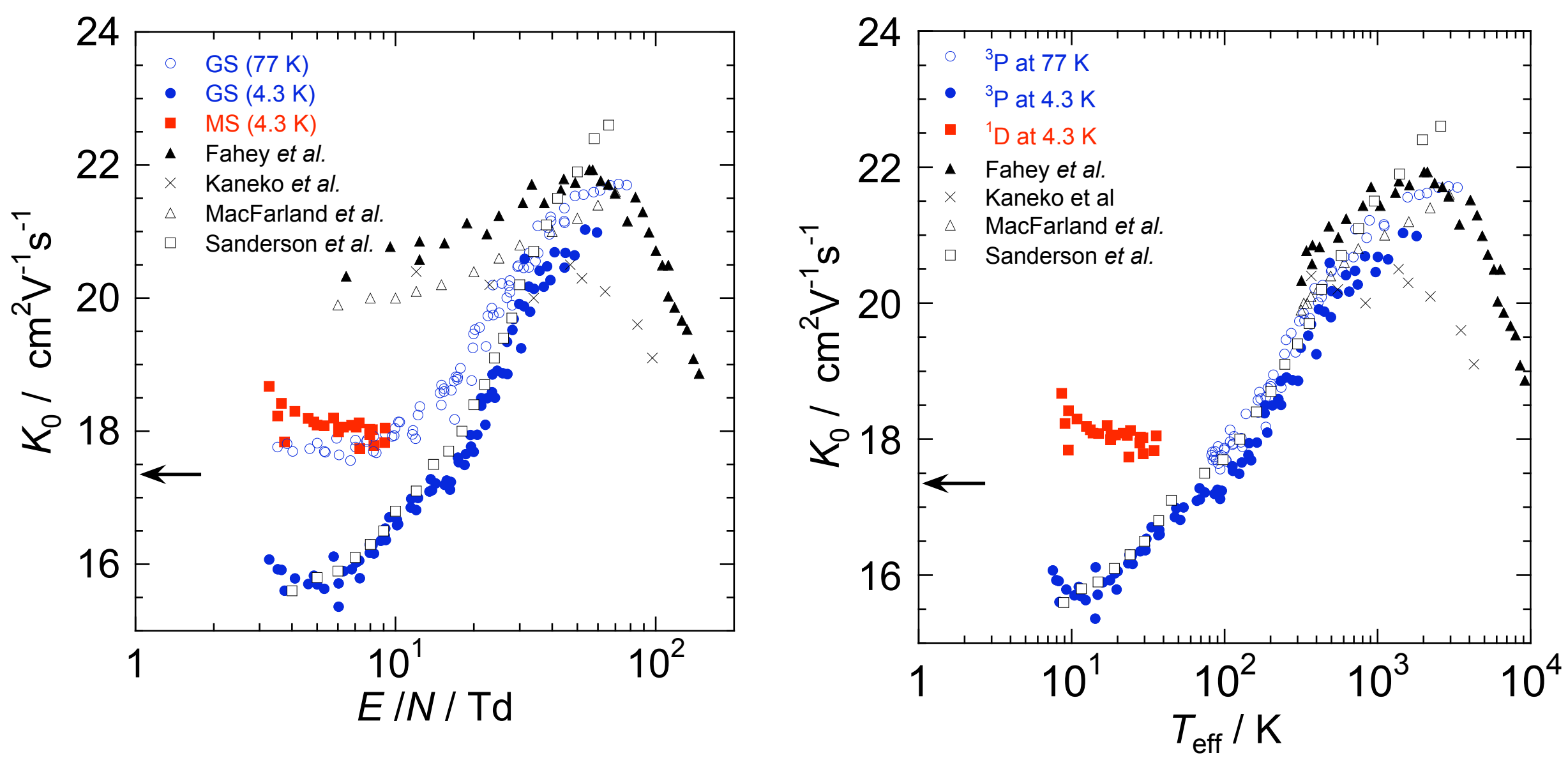

$K_{0}(\mathrm{MS})>K_{\text {pol }}>K_{0}(\mathrm{GS})$ in $\mathrm{N}^{+} / \mathrm{He}$ 


\section{Potential curves and Mobility of $\mathrm{N}^{+}$in $\mathrm{He}$}
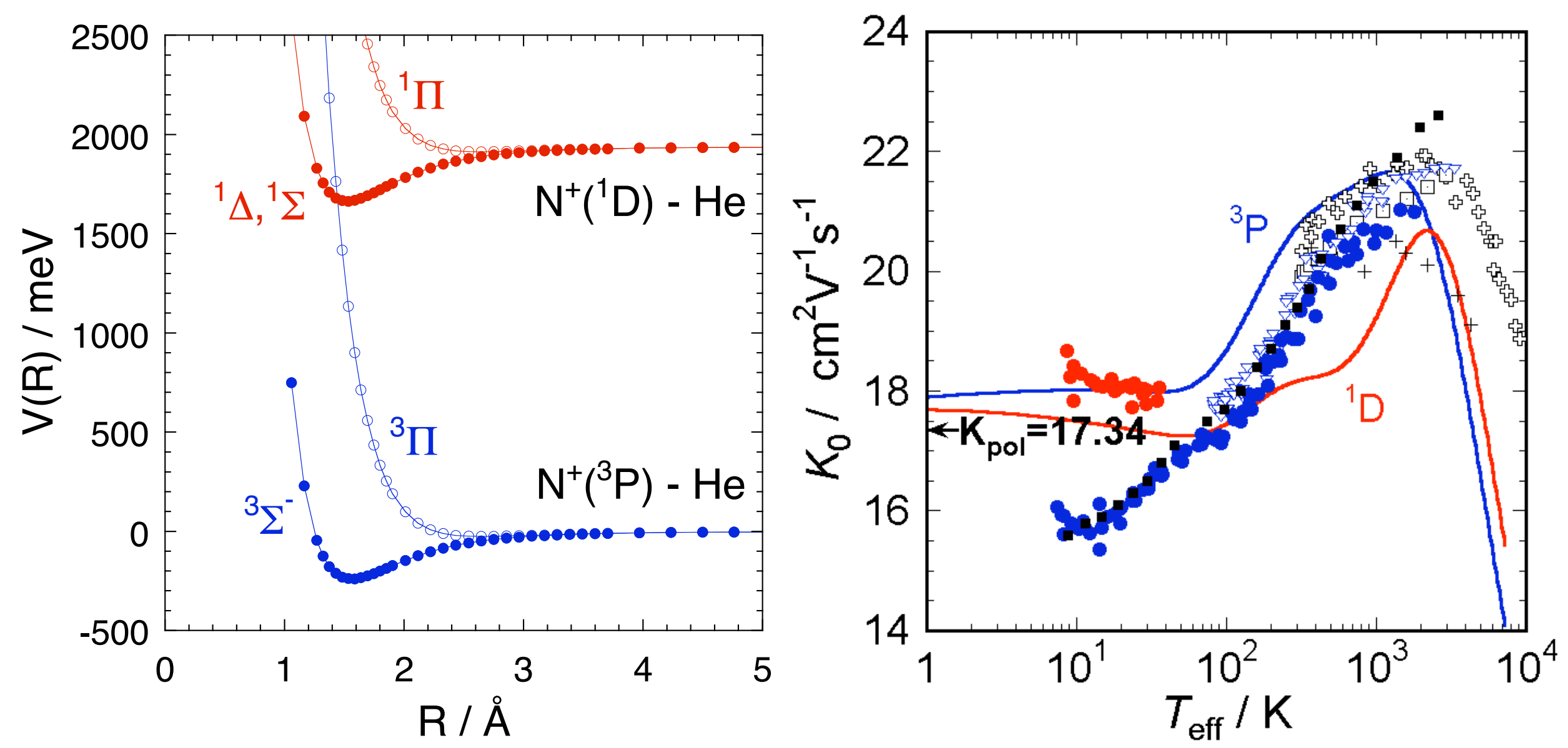

Mobility of ${ }^{1} \mathrm{D}$ state can be explained, but that of ${ }^{3} \mathrm{P}$ is not. 


\section{Summary}

Close-shell systems : Alkali ions

- We measured the mobility of five alkali ions in cooled He.

- The results are explained by the calculation with qunantal cross sections taking account one molecular state.

Open-shell systems : $\mathrm{C}^{+}$and $\mathrm{N}^{+}$ions

1. We measured the mobility of ions in the ground and excited (metastable) states

2. The calculation with classical cross sections concerning two molecular states can explain the experimental results on $\mathrm{C}^{+}\left({ }^{4} \mathrm{P}\right.$ and $\left.{ }^{2} \mathrm{P}\right)$ and $\mathrm{N}^{+}\left({ }^{1} \mathrm{D}\right)$ in $\mathrm{He}$.

3. Mobility of $\mathrm{N}^{+}\left({ }^{3} \mathrm{P}\right)$ cannot be explained by our calculation. This is an open question. 


\section{Do you need mobility data?}

